\title{
Modeling the Photocatalytic Process of Variation in Chemical Oxygen Demand via Stochastic Differential Equations
}

\author{
Adriano F. Siqueira*, Oswaldo L. C. Guimarães, Hélcio J. Izario Filho, Domingos S. Giordani, Ivy \\ dos Santos Oliveira, Henrique Otávio Queiroz de Aquino and Messias Borges Silva
}

\author{
Engineering School of Lorena- EEL - University of São Paulo C. Postal 116-CEP:12.602-810 - Lorena-SP , Estrada \\ Municipal do Campinho, $s / n^{o}$ Brazil
}

\begin{abstract}
Several papers in the literature on Advanced Oxidation Processes (AOPs) confirm the process as a viable alternative for the treatment of a variety of industrial effluents. In many of these works, modeling the variations of Chemical Oxygen Demand (COD) as a function of different experimental conditions was performed by techniques such as Design of Experiments, Artificial Neural Networks and Multivariate Analysis. These techniques require both a large number of parameters and a large quantity of experimental data for a systematic study of the model parameters as a function of experimental conditions. On the other hand, the study of Stochastic Differential Equations (SDE) is presently well developed with several practical applications noted in the literature. This paper presents a new approach in studying the variations of COD in AOPs via SDE. Specifically, two effluents, from the manufacture of paints and textiles were studied by combined treatment of the photo-Fenton process and catalytic ozonization.
\end{abstract}

Keywords: Modeling, Stochastic Differential Equations, Chemical Oxygen Demand, Photo-Fenton process, Ozonization.

\section{INTRODUCTION}

Chemical oxygen demand is an important parameter for estimating the concentration of organic contaminants in water supplies and industrial wastes. Since the degradation of organic compounds demand oxygen, the concentrations of these substances can be estimated by the amount of oxygen required. A method using dichromate as the oxidizing agent in a closed system is critical in determining COD due to dichromate's high oxidation potential and its operational ease for a wide variety of sample types. Inorganic species such as the $\mathrm{O}_{2}{ }^{2-}, \mathrm{Fe}^{2+}$, halogens and $\mathrm{SO}_{2}$ have a reduction capacity, especially for potassium dichromate, interfering positively in test results. Also, the interference of chloride and nitrite is preventable by the addition of mercuric sulfate and sulfamic acid, respectively. However, a method correcting the interference of inorganic species such as $\mathrm{Fe}^{2+}$ and $\mathrm{H}_{2} \mathrm{O}_{2}$ is not mentioned in standard methods and is also poorly reported in the literature [1].

Several industrial effluents are resistant to degradation by conventional processes such as biological or physicalchemical. Thus, advanced chemical oxidation processes (AOPs) such as $\mathrm{H}_{2} \mathrm{O}_{2} / \mathrm{UV}, \mathrm{O}_{3}$ and Fenton's reagent are very promising techniques in industrial applications [2].

Advanced chemical oxidation processes are divided into two groups: homogeneous and heterogeneous. The first

*Address correspondence to this author at the Engineering School of Lorena- EEL - University of São Paulo C. Postal 116-CEP:12.602-810 Lorena-SP , Estrada Municipal do Campinho, $\mathrm{s} / \mathrm{n}^{\circ}$ Brazil;

Tel: 55123159 5089; E-mails: adriano@debas.eel.usp.br;

oswaldocobra@debas.eel.usp.br occurs in one step and uses ozone, hydrogen peroxide or Fenton's reagent (a mixture of $\mathrm{H}_{2} \mathrm{O}_{2}$ with $\mathrm{Fe}^{2+}$ salt) as generators of hydroxyl radicals. The second type uses semiconductors as catalysts (titanium dioxide, zinc oxide, etc.) [3]. The use of UV radiation and the semiconducting properties of the catalyst allow for the formation of hydroxyl radicals and subsequent oxidation of effluent.

Besides inorganic species present in effluent, which can interfere with the measurement of COD, there is the additional presence of the residual oxidizing agent $\mathrm{H}_{2} \mathrm{O}_{2}$. Typically, hydrogen peroxide is unstable in natural conditions (temperature: $25 \pm 2{ }^{\circ} \mathrm{C}$ ) and degrades rapidly in oxygen and water due to the existence of organic and inorganic catalysts. The leading factors contributing to the decomposition of $\mathrm{H}_{2} \mathrm{O}_{2}$ are an increase of temperature and $\mathrm{pH}$ (especially at $\mathrm{pH}$ above 6-8), the presence of contaminants (mainly transition metals such as copper, manganese and iron) and a lowimportance exposure to ultraviolet radiation. In most cases, $\mathrm{pH}$ and contaminants are predominant factors in the process of $\mathrm{H}_{2} \mathrm{O}_{2}$ decomposition. Thus, the production of hydroxyl radicals is influenced by several factors providing random behaviors in degradation results.

Neyens and Bayens [4] observed an increase in the value of COD after the addition of $\mathrm{H}_{2} \mathrm{O}_{2}$. They concluded that this variation was due to the presence of unconsumed oxidant residues in testing causing a positive interference in the analysis. The presentation of high concentrations of residual peroxide should be removed from the solution. The excess of $\mathrm{H}_{2} \mathrm{O}_{2}$ may also have contributed to the sequestration of hy- 
droxyl radicals through the reaction between hydroxyl radical and $\mathrm{H}_{2} \mathrm{O}_{2}$, thus reducing the efficiency of degradation.

Several researchers [5] and [6] reported the significant interference of $\mathrm{H}_{2} \mathrm{O}_{2}$ in the measurement of COD.

Potassium dichromate $\left(\mathrm{K}_{2} \mathrm{Cr}_{2} \mathrm{O}_{7}\right)$, a powerful oxidant, can be used for the analysis of COD. Excessive amounts of hydrogen peroxide will react with potassium dichromate and, due to the presence of another oxidant, act as a reducing agent. This changes the value of COD experimentally to a false higher value.

Random COD values, apart from measurement errors, can also be derived from reaction conditions. One example is the system $\mathrm{Fe}^{2+} / \mathrm{Fe}^{3+}-\mathrm{H}_{2} \mathrm{O}_{2}$, which has a maximum catalytic activity between $\mathrm{pH} 2.8$ and 3.0. A decrease in $\mathrm{pH}$ depresses catalytic activity consequently inhibiting the complexation of $\mathrm{Fe}^{3+}$ with $\mathrm{H}_{2} \mathrm{O}_{2}$. Iron precipitates flakes can form at higher $\mathrm{pH}$ values.

This paper presents the degradation process modeling for two effluents via homogeneous oxidation processes based on the action of the reagent $\mathrm{H}_{2} \mathrm{O}_{2}$ with chemical oxygen demand as response variable.

The objective is to propose a model for the measurement of COD as a function of reaction time in an advanced oxidation processes via photo-Fenton process and catalytic ozonization using stochastic differential equations. This allows for the modeling of the statistical mean value and also the dispersion of data. By this model it is possible to construct confidence intervals that allow for the evaluation of intrinsic interference in the analysis of each experimental point. Moreover, it is possible to perform simulations of conversion versus time duration under experimental conditions and in consideration of the variability of experimental data.

Our paper first presents the definition of the model's properties and parameter estimators. Afterwards, the model is adjusted for the two types of effluent treated by AOPs. For the first effluent, the model was adjusted to seven different experimental conditions with the aim of investigating how the parameters of the stochastic equation vary per condition. The capacity for the model to show the influence of experimental conditions on the conversion of COD as a function of time was, likewise, investigated. However, a systematic study of operating-condition influences on the model parameters is not addressed in this paper. Finally, the model's applicability to an alternate effluent treated by another AOP is verified for degradation reactions in different systems.

\section{CASE STUDIES - APPLICATION OF ADVANCED OXIDATION PROCESSES}

Advanced Oxidation Processes are a technology potentially capable of degrading recalcitrant effluents. Both the study and refinement of technical applications is essential in facilitating the optimization of such processes.

Hydroxyl radicals produced in the oxidation reaction can attack organic molecules by the abstraction of a hydrogen atom from the molecule of the compound to be treated. Equations 1 to 4 present the degradation sequence where $R$ is an organic compound.

$$
\begin{aligned}
& \mathrm{OH}^{\bullet}+\mathrm{RH} \rightarrow \mathrm{H}_{2} \mathrm{O}+\mathrm{R}^{\bullet} \\
& \mathrm{R}^{\bullet}+\mathrm{H}_{2} \mathrm{O}_{2} \rightarrow \mathrm{ROH}+\mathrm{OH}^{\bullet} \\
& \mathrm{R}^{\bullet}+\mathrm{O}_{2} \rightarrow \mathrm{ROO} \\
& \mathrm{ROO}^{\bullet}+\mathrm{RH} \rightarrow \mathrm{ROOH}+\mathrm{R}^{\bullet}
\end{aligned}
$$

Fenton's reagent was discovered approximately 100 years ago. Its application as an oxidation process agent to destroy organic compounds dates from 1960. The Fenton reaction has the advantage of completely destroying contaminants and producing water, carbon dioxide and inorganic salts by the dissociation of the oxidant and the formation of hydroxyl radical.

Fenton's reagent is characterized as a mixture of hydrogen peroxide and iron salts generating hydroxyl radicals. This is seen in Equations 5 and 6 [4].

$$
\begin{aligned}
& \mathrm{Fe}^{+2}+\mathrm{H}_{2} \mathrm{O}_{2} \rightarrow \mathrm{Fe}^{+3}+\mathrm{OH}^{\bullet}+\mathrm{OH}^{-} \\
& \mathrm{OH}^{\bullet}+\mathrm{Fe}^{+2} \rightarrow \mathrm{OH}^{-}+\mathrm{Fe}^{+3}
\end{aligned}
$$

Equation 5 describes the initiation reaction for the Fenton process and Equation 6 shows the reaction termination.

Hydroxyl radical production can be increased by the application of ultraviolet radiation as shown in Equation 7.

$\mathrm{Fe}(\mathrm{OH})^{2+}+h v \rightarrow \mathrm{Fe}^{2+}+\cdot \mathrm{OH}$

This combination of the Fenton reaction with ultraviolet is known as a photo-Fenton reaction. The concentration of $\mathrm{H}_{2} \mathrm{O}_{2}$ is a critical factor in this process, blocking or reducing the subsequent consumption of $\mathrm{H}_{2} \mathrm{O}_{2}$ and requiring the addition of more $\mathrm{H}_{2} \mathrm{O}_{2}$. Excessive $\mathrm{H}_{2} \mathrm{O}_{2}$ can also act as sequestering agent of hydroxyl radicals to form the hydroperoxyl radical ${ }^{\circ} \mathrm{O}_{2} \mathrm{H}$ that has a lower reduction potential $\left(\mathrm{E}^{\circ}=1.42 \mathrm{~V}\right)$ than hydroxyl ${ }^{\bullet} \mathrm{O}_{2} \mathrm{H}$, impacting the degradation process [7].

Alternatively, ozone is occasionally produced and decomposed by UV photo-degradation when a resultant oxygen atom reacts with water producing ${ }^{\circ} \mathrm{OH}$ in the intermediate production of photolyzed hydrogen peroxide (Equations 8 and 9).

$$
\begin{aligned}
& \mathrm{O}_{3} \stackrel{\mathrm{UV}}{\longrightarrow} \mathrm{O}_{2}+\mathrm{O} \\
& \mathrm{O}+\mathrm{H}_{2} \mathrm{O} \rightarrow \mathrm{H}_{2} \mathrm{O}_{2} \stackrel{U V}{\longrightarrow} 2^{\circ} \mathrm{OH}
\end{aligned}
$$

A fraction of oxygen atoms produced by the photolysis of ozone is electronically excited and reacts with water to produce hydroxyl radicals.

The oxidation of organic compounds dissolved in water from ozonation can be considered as an AOP. In fact, hydroxyl radicals can be generated from the decomposition of ozone in the presence of hydroxyl ions and/or initiated by the trace presence of other substances such as transition metal cations. Basically, the process of ozonation can take two preferred paths: 1) a direct path through a molecular ozone reaction, or 2) by a radical path resulting from the reaction between hydroxyl radicals generated by ozone decomposition $[8]$. 
The combinative use of ozone or hydrogen peroxide with UV processes offers greater advantages over separate individual applications in light of the significant increase in the ratio of hydroxyl radical formations.

Ozone is a powerful oxidant $\left(\mathrm{E}^{\circ}=2.07 \mathrm{~V}\right)$, which can react with unsaturated molecular bonds $(\mathrm{C}=\mathrm{C}, \mathrm{C}=\mathrm{N}, \mathrm{N}=\mathrm{N}$, etc.). The generation of hydroxyl radicals enhances the ability to attack these compounds by the combined use of hydrogen peroxide.

The main advantage of using combined processes for water treatment is the synergism between photolysis and hydrogen peroxide allowing the degradation of compounds that require elevated oxidizing power [9].

\subsection{Materials and Methods}

Experimental data for the study of the model were obtained from the treatment of two types of industrial effluents:

- Compounds from various paint industries formed a mixture with a COD of approximately $240000 \mathrm{mg} \mathrm{L}^{-1}$. These effluents from the manufacture of polyester and alkyd resins have high organic load and hence high value of COD

- Effluents from textile industry are highly variable in composition and contain various combinations of raw materials and intermediate products. This effluent has high organic load, strong color (dyes) and also high concentrations of dissolved solids. Due to the elevated variability in effluents, the textile sector actively pursues new treatment technologies [2].

\subsection{Effluent from the Manufacture of Polyester and Al- kyd Resins}

Samples were homogenized and processed into a "final sample" which was used for analysis and stored at $5{ }^{\circ} \mathrm{C}$ to minimize the possible changes of initial conditions. The sample was characterized after mixing as an effluent with high concentrations of pollutants due to a COD value of approximately $240000 \mathrm{mgL}^{-1}$.

A Pyre ${ }^{\circledR}$ glass reactor of $1000 \mathrm{~mL}$ of capacity equipped with stirring system and ozone diffuser was utilized as the containment vessel. Additional equipment included a temperature-controlled thermostatic bath, ozonator and an ultraviolet (UV) source of two mercury vapor lamps at $125 \mathrm{~W}$ each.

An iron sulfate solution $\left(0.18 \mathrm{~mol} . \mathrm{L}^{-1}\right)$ and a hydrogen peroxide solution at concentration of $30 \%$ (by weight) were used initially.

\subsection{Effluent from Textile Industry}

Experiments in AOP treatment were performed in a tubular photochemical reactor (Model FPG-463/1) with a nominal volume of approximately $1 \mathrm{~L}$, equipped with lowpressure mercury lamp model-GPH 463T5L emitting UV radiation at $254 \mathrm{~nm}$ at $28 \mathrm{~W}$ and protected by a quartz tube. The liquid temperature was controlled by a thermostatic bath UNITEMP (Model 112D Fanem). The circulation of the effluent from the tubular reactor to the storage tank was per- formed using a low-power centrifugal pump. Ozone was generated by the conversion of $\mathrm{O}_{2}$ into $\mathrm{O}_{3}$ using AUJE, Model MV 2001 by electric discharge over dielectric barriers (Corona effect) at $220 \mathrm{~V}$, a maximum of $60 \mathrm{~W}$ and pressure below 2 bar. Photochemical treatment was performed in a batch process. The Fenton reagent $\left(\mathrm{H}_{2} \mathrm{O}_{2}\right.$ at $30 \%$ by weight and $\mathrm{FeSO}_{4} \cdot 7 \mathrm{H}_{2} \mathrm{O} 0$ at 18 mol. $\mathrm{L}^{-1}$ ) was added to the reaction system using two burettes by dripping $\mathrm{H}_{2} \mathrm{O}_{2}$ first with subsequent additions of $\mathrm{FeSO}_{4} \cdot 7 \mathrm{H}_{2} \mathrm{O}$.

Thus the experimental data derived for mathematical modeling were from two different effluents treated by separate oxidation processes.

\section{MATHEMATICAL MODEL}

Consider the functions $f$ and $g$ such that:

$$
f, g:[0, T] \times R \rightarrow R
$$

Also consider $W(t)$ in the Wiener Process, which is a mathematical description of Brownian motion proposed by Norbet Wiener. Durrett [10] defined the Wiener Process as a continuous Gaussian process with independent increments where: $E\{W(t)\}=0 \quad$ and $\operatorname{Var}\{W(t)-W(s)\}=t-s, \quad \mathrm{t}>\mathrm{s}$. Furthermore, the probability distribution of $W(t)-W(s)$ is a normal distribution with zero mean and variance $t-s$. Our work utilized the Itô stochastic differential equation shown in Equation 10.

$$
d X_{t}=f\left(t, X_{t}\right) d t+g\left(t, X_{t}\right) d W_{t}
$$

According to Klebaner [11], function $f$ can be understood as a measure of the overall tendency of $X_{t}$ and $g$ as the measure of standard deviation for variations. If the functions $f$ and $g$ satisfy certain continuity conditions, as per [10] and [11], $X_{t}$ presents a unique solution.

The empirical SDE proposed to study the variation of COD in this paper is given by Equation 11 .

$$
d X_{t}=\left(a+\frac{b k}{e^{k t}}\right) d t+\frac{c}{(t+1)^{p}} d W_{t}
$$

Where $a, b, c, k$ and $p$ are model parameters dependent on experimental conditions. $X_{t}$ is the conversion of COD at time $t$, in minutes. The heuristic for this proposal can be found in the properties of Equation (11). It is not difficult to show that this equation has the following properties:

a) For each $t>0$, it can be proved, [11], that $X_{t}$ has a normal distribution with mean and variance shown in Equations 12 and 13:

$$
\begin{aligned}
& E\left(X_{t}\right)=a . t+b\left(1-e^{-k . t}\right)=\mu_{t} \\
& \operatorname{Var}\left(X_{t}\right)=\frac{c^{2}}{2 p-1}\left(1-\frac{1}{(t+1)^{2 p-1}}\right)=\sigma_{t}^{2}
\end{aligned}
$$

b) The covariance between $X_{t}$ and $X_{s}$, to all $t>s$.

$E\left\{\left[X_{t}-E\left(X_{t}\right)\right]\left[X_{s}-E\left(X_{s}\right)\right]\right\}=\operatorname{Var}\left(X_{s}\right)$

c) From property $(b)$ it is known that the correlation coefficient between $X_{t}$ and $X_{s}$ for $t>s>0$ can be calculated as in Equation 14. 
$\rho_{s t}=\frac{\sigma_{s}}{\sigma_{t}}$

Note that in Equation $13 \sigma_{t}$ is greater than $\sigma_{s}$ to $t>s$. The result (Equation 14) indicates that the data generated by the model for large time durations show significant correlation. In fact, this behavior was observed in all experiments and the linear trend observed from approximately $20 \mathrm{~min}$ of reaction.

d) $X_{t}$ is a Gaussian process, i.e., the joint distribution of a finite number of members $X_{t 1}, X_{t 2}, \ldots X_{t \mathrm{tn}}$ in a multivariate normal vector with average, $E(X)$ and covariance matrix $\operatorname{Cov}\left(X_{t i}, X_{t j}\right)=\operatorname{Var}\left(X_{t i}\right)$, property $(d)$ given, respectively, by Equations 16 and 17.

$E(X)=\left(\mu_{t 1}, \mu_{t 2}, \ldots, \mu_{t n}\right)$

$\Sigma=\left[\begin{array}{cccc}\sigma_{t 1}^{2} & \sigma_{t 1}^{2} & \ldots . . & \sigma_{t 1}^{2} \\ \sigma_{t 1}^{2} & \sigma_{t 2}^{2} & \ldots . . & \sigma_{t 2}^{2} \\ \vdots & \vdots & \vdots & \vdots \\ \sigma_{t 1}^{2} & \sigma_{t 2}^{2} & \ldots . . & \sigma_{t n}^{2}\end{array}\right]$

This property can be proved based on [11]. Subsequently, the variables observed in the process $X_{t 1}, X_{t 2}, \ldots X_{t \mathrm{n}}$, are not independent and therefore there is a correlation structure between them evidenced by the correlation matrix.

In Equation 12, when $k$ is large, shows that the average shape of the curve exhibits elevated exponential growth, stabilizing at a plateau in the value of the parameter $b$. The speed of this growth is controlled by the constant $k$. After reaching this plateau, the expected value presents linear behavior modeled by the term a.t. In all the experimental runs analyzed, the value of the parameter is minimal and in many cases zero based on the confidence interval of the parameter. Thus, for our model, it was possible to approach a reaction time of nearly $99 \%$ of the plateau with the following simple equation:

tpat $=\frac{2 \cdot \ln (10)}{k}$

Equation 18 reinforces the interpretation that the parameter $k$ is related to the reaction speed reaching a plateau.

From the properties in Equation 13, it can be observed that the variability in the initial time of $X_{t}$ is zero. This agrees with the initial conversion to be zero. Moreover, the variance of $X_{t}$ grows until stabilized at a value dependent on constants $c$ and $p$. This behavior was also evident in the experimental runs analyzed from different types of effluent treated with AOPs. The growth rate of variability depends on the constant $p$. Finally the presence of Brownian motion is justified by existence of interference phenomenon between the reactants of the AOP leading to errors in concurrent COD readings, as discussed in the introduction.

\subsection{Estimators}

According to property (a) parameters $c$ and $p$ are related to data dispersion and were estimated by the technique used by [5]. With that property, from discrete experimental observations of each experimental run and from the stochastic model of the equation, the authors present estimators for SDE parameters. Applying this technique, it is possible to obtain an estimator to $c$, as shown in Equation 19:

$c=\frac{1}{20} \sqrt{\frac{\sum Q_{i}}{\sum \frac{1}{\left(t_{i}+1\right)^{2 p}}}}$

where: $Q_{i}$ is calculated as:

$Q_{i}=\frac{\left(\Delta X_{i}\right)^{2}}{\Delta t_{i}}$

The calculation of $Q_{i}$ necessitates finding the squared value of the $X_{i}$ conversion variation divided by the respective time variation. Thus, from the variability of all experimental data, in each experimental run, a different parameter $c$ estimative will be found.

However, an estimation of the value of $c$ in Equation 19 requires finding the value of $p$. An estimate for $p$ can be obtained from the numerical solution of the Equation 21.

$$
\frac{\sum Q_{i}}{\sum \frac{1}{\left(t_{i}+1\right)^{2} p}}-\frac{\sum P_{i}}{\sum \frac{4 X_{i}^{2}}{\left(t_{i}+1\right)^{2} p}}=0
$$

where $P_{i}$ is given in Equation 22 :

$P_{i}=\frac{\left(\Delta X_{i}^{2}\right)^{2}}{\Delta t_{i}}$

Thus, $p$ is the value rendered in Equation 21 and tending towards zero or result in a value within an acceptable error limit (an order of magnitude of $10^{-2}$ in this work). Several experiments on the first effluent, conducted with different values of temperature, $\mathrm{pH}$ and concentrations of oxidizing reagents, produced a confidence interval value of $95 \%$ for average alpha of $0.46 \pm 0.01$.

As previously noted, parameters $a, b$ and $k$ are related to the shape of the mean curve. Although it is possible to use the method of [12] for estimating these parameters, the result is a nonlinear system with three equations difficult to solve. A simpler alternative to estimate these parameters was adopted in this work in the iterative algorithm described below.

Algorithm (1):

a) Assign initial value for $k$, (in all reactions examined in this study $k$ was between 0 and 1 );

b) with $k$ fixed, the values of $a$ and $b$ can be estimated by a least squares method using Equation 12;

c) Calculate the quadratic sum of the average value of the model, as per Equation 12, using our experimental data. This value represents a measure of how accurate the average model is in relation to the experimental data;

d) Repeat the algorithm for different values of $k$ and adopt the result that produces a lower value for the quadratic 
sum in Equation 12 in non-decreasing form. This condition is important based on an expectation of the average value of conversion increasing with reaction duration.

\subsection{Numerical Simulation of a SDE}

The easy-to-implement Euler-Maruyama algorithm was used in this work to obtain numerical simulations of Equation 11. A detailed study of the EDS numerical simulations can be found in [13].

Equation (11) can be discretized as follows:

$$
X_{n+1}=X_{n}+\left(a+\frac{b \cdot k}{e^{k . t_{n}}}\right) \Delta t_{n}+\frac{c}{\left(t_{n}+1\right)^{p}} \Delta W_{n}
$$

In Equation $23 \Delta W_{n}$ represents the increment of Wiener process calculated by a normal distribution with zero mean and variance $\Delta \mathrm{t}_{\mathrm{n}}$. Note that in Equation 23, all necessary information to estimate the value of $X_{(\mathrm{n}+1)}$ depends only on the information at time $t n$. In all simulations the value of $\Delta t_{n}$ was 0.01 . All simulations were performed with Matlab.

\subsection{Confidence Intervals}

The confidence intervals are an important tool for estimating the parameters and to study the variability of the process $X_{t}$. Based on the property (a) for each time $t$, one can calculate a confidence interval with $95 \%$ confidence for the process $X_{t}$ in Equation 24.

$E\left(X_{t}\right) \pm 1.96 * \mathrm{dp}\left(X_{t}\right)$

Where $\mathrm{dp}\left(X_{t}\right)$ is the square root of the Equation 13 results.

An estimate for the error can be obtained for parameter $p$ through the error limit of Equation 21. A confidence interval of $95 \%$ adopted, for the least squares estimators found in Equation 13, for parameters $a$ and $b$, since the $k$ value found by the algorithm described above. Finally, the study of the variability for parameter $k$ was examined in numerical simulations of Equation 11. This technique is known as a parametric bootstrap [14].

The algorithm (2) below was used for the computational calculation of the confidence interval for parameter $k$ :

a. from an experimental run to estimate the parameters $\hat{\mathrm{A}}=$ $(a, b, c, k, p)$ as previously described;

b. in Equation 23, the estimated parameters $(\hat{\mathrm{A}})$, generate a stochastic simulation and select the values of simulated $X_{t}$ for time intervals of 10 minutes. The experimental procedure also collected information in 10 minute intervals. These collected values are called bootstrap replicas and are considered as a new experimental run performed under the experimental conditions of the run under analysis. Again, using the procedure of work, estimate the parameters $\mathrm{A}^{*}=\left(a^{*}, b^{*}, c^{*}, k^{*}, p^{*}\right)$ and repeat the procedure several times for each experimental run culminating in 200 replications in this work;

c. and, finally, with a histogram of 200 values for $k^{*}$, is possible to obtain a confidence interval for $k$.

\section{RESULTS AND DISCUSSIONS}

Initially, Fig. (1) illustrates the ability of the stochastic model to simulate the experimental behavior of the reactions to the alkyd effluent sample (Effluent 1). In this figure, the red line represents the average model values with magenta lines indicating a confidence interval of $95 \%, E\left(X_{t}\right) \pm$ $1.96 \cdot \mathrm{dp}\left(X_{t}\right)$, for conversion values in each $t$ instant of time. Moreover, the result of the numerical simulation of the stochastic equation (in blue), showed the random behavior of the model. The average value of the model shows a behavior consistent with the trend of experimental data. Regarding the dispersion value, with the confidence interval of $95 \%$, there is a good fit between the variability of the experimental data and the model. In this experimental run, most of the data shows small fluctuations around the average model, which is shown by stochastic simulation (in blue). Otherwise, three experimental points near the edge of the confidence interval were presented, indicating a low probability that these points were generated by the model. This may indicate the presence of high values of interference and/or experimental errors in measurement. Another important indicator given by the confidence interval is that a repetition of the reaction, with the same experimental conditions, will generate conversion rates that, in all likelihood, will be within the magenta interval in Fig. (1).

Characteristic of these reactions was the presence of a rapid conversion variation for the initial instants, approximately the first 20 minutes, followed by a plateau in the remainder of the reaction duration. The results of seven experimental runs on effluent from a manufacture of polyester and alkyd resins are presented in Table 1 defining the model's capacity for articulating experimental conditions influencing the conversion rate in early stages. Table $\mathbf{2}$ shows estimates for all model parameters (2) in each of the experimental runs in Table 1 . The last column of Table $\mathbf{2}$ shows the $\mathrm{R}^{2}$ of the average value for the model indicating the proximity of this function respective to the experimental data. Fig. (2) shows the commonalities of the model with the parameters of Table 2.

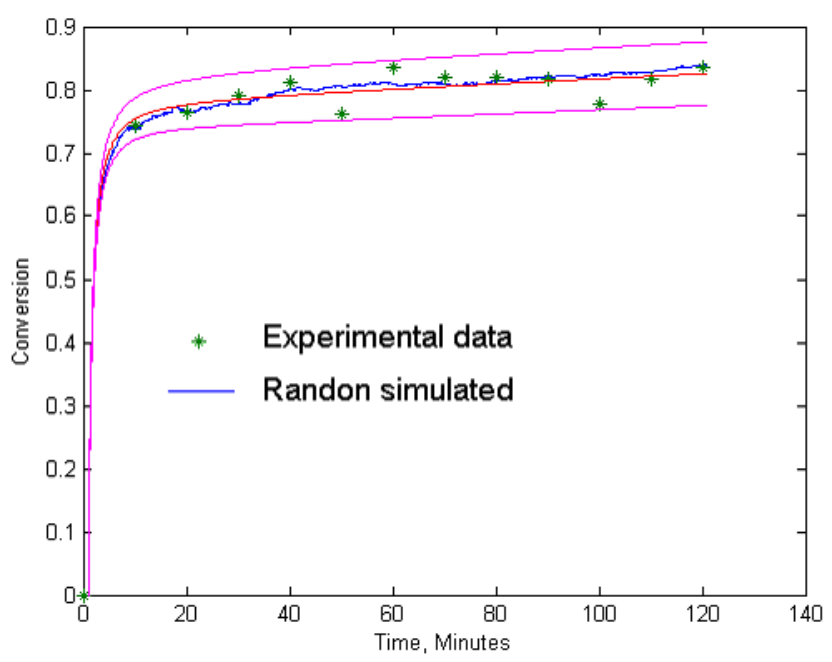

Fig. (1). Numerical simulation of SDE (11) with parameters obtained in the experimental condition 7 of Table 1. 
Table 1. Experimental Conditions Analyzed for Polyester and Alkyd Resin Effluent

\begin{tabular}{|c|c|c|c|c|c|c|}
\hline $\mathbf{N}^{\circ}$ & $\mathbf{U V}$ & $\mathbf{p H}$ & $\mathbf{T}\left({ }^{\circ} \mathbf{C}\right)$ & $\mathbf{H}_{2} \mathbf{O}_{2}$ & $\mathrm{Fe}^{2+}$ & $\mathbf{O}_{3}$ \\
\hline 1 & 1 & 2 & 25 & 25 & 26 & 4 \\
\hline 3 & 0 & 2 & 30 & 19 & 20 & 2 \\
\hline 4 & 1 & 3 & 30 & 25 & 26 & 0 \\
\hline 6 & 1 & 2 & 35 & 19 & 20 & 2 \\
\hline 7 & 1 & 3 & 35 & 19 & 20 & 4 \\
\hline
\end{tabular}

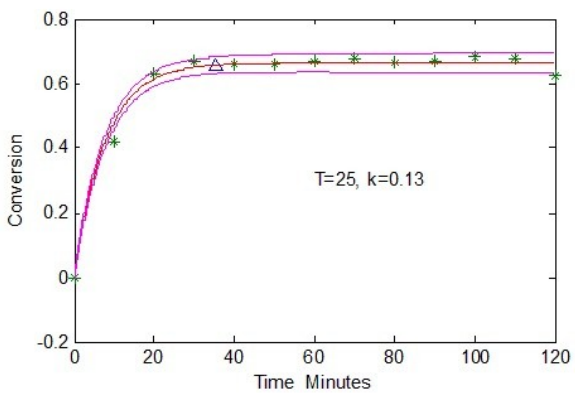

(2a)

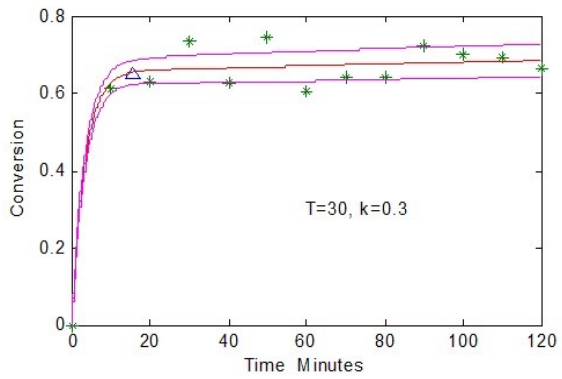

(2d)

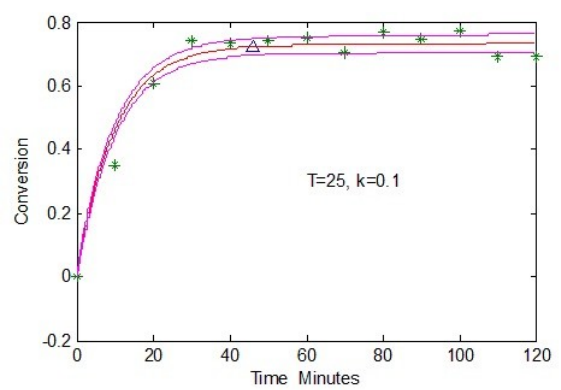

(2b)

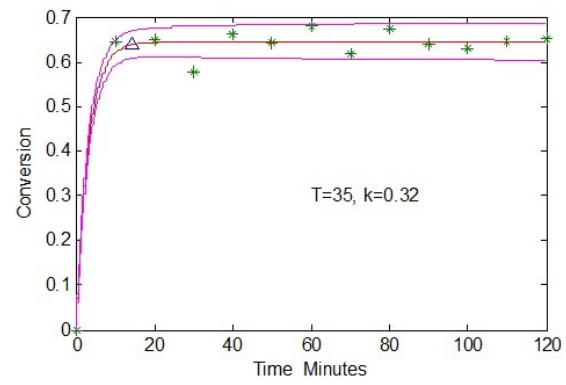

(2e)

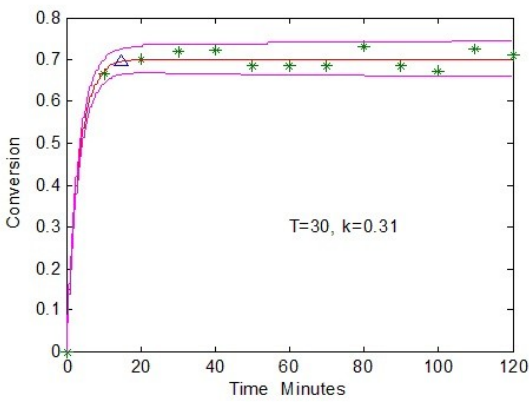

(2c)

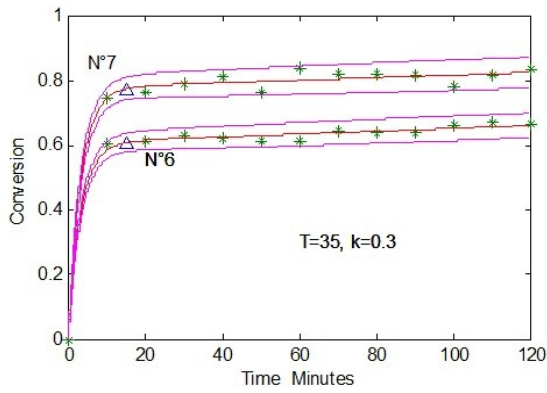

(2f)

Fig. (2). (a-f). Manufacturer's polyester and alkyd resin effluent treated under the conditions of Table 1. The triangle marks the estimated time to reach the plateau obtained by equation (18).

With the aid of confidence intervals, it can be observed that there was no significant difference in the values of $k$ at temperatures of 30 and $35{ }^{\circ} \mathrm{C}$ under the experimental conditions. Therefore, as shown in Equation 18, there is no difference in the estimated time to reach a plateau. This fact is shown clearly in the items from (a) to (f) in Fig. (2) where the time of each reaction to reach its respective plateau is shown with a triangle mark. However, at $25^{\circ} \mathrm{C}$, the model indicates that the concentrations of reagents have an influence on the parameter $k$.

Comparing items (a) and (b) with the other items in Fig. (2) for parameter $k$ shows evidence for faster reaction rates in the initial instants and, consequently, less time spent reaching a plateau.
An interesting comparison can be done between reactions 2 and 7. The experimental conditions of the two reactions are the same, except that the reaction 7 temperature is $35{ }^{\circ} \mathrm{C}$ while reaction 2 occurs at $25{ }^{\circ} \mathrm{C}$. From the analysis of parameter $b$ indicating the plateau of reaction, there is no evidence that these plateaus are different. However, the times to reach these levels are significantly different according to the analysis of the parameter $k$. This analysis confirms that higher temperatures result in a faster attainment of the plateau under experimental condition. An analysis of parameter $a$ shows evidence that the higher temperature of the seventh reaction influenced the slope of the plateau to be different from zero. This indicates that the degradation reaction remains significant after the reaction reaches a plateau. Under our experimental conditions, this occurred at a temperature of $35{ }^{\circ} \mathrm{C}$ in the presence of UV and concentrations of $\mathrm{H}_{2} \mathrm{O}_{2}$ 
Table 2. Model Parameters Obtained from Analyzed Conditions Polyester and Alkyd Resin Effluent

\begin{tabular}{|c|c|c|c|c|c|c|}
\hline $\mathbf{N}$ & $\mathbf{a}$ & $\mathbf{b}$ & c & $\mathbf{p}$ & $\mathbf{k}$ & $\mathbf{R}^{2}$ \\
\hline 1 & $0.0000+/-0.0005$ & $0.66+/-0.04$ & $0.007+/-0.002$ & 0.46 & $0.130+/-0.007$ & 0.9822 \\
\hline 2 & $0.0000+/-0.0009$ & $0.73+/-0.07$ & $0.006+/-0.003$ & 0.46 & $0.100+/-0.004$ & 0.9522 \\
\hline 3 & $0.0000+/-0.0004$ & $0.70+/-0.03$ & $0.009+/-0.002$ & 0.44 & $0.31+/-0.04$ & 0.9883 \\
\hline 4 & $0.0002+/-0.0008$ & $0.66+/-0.06$ & $0.009+/-0.005$ & 0.47 & $0.30+/-0.05$ & 0.9362 \\
\hline 5 & $0.0000+/-0.0005$ & $0.64+/-0.04$ & $0.009+/-0.003$ & 0.45 & $0.32+/-0.06$ & 0.9729 \\
\hline 6 & $0.0004+/-0.0002$ & $0.61+/-0.02$ & $0.008+/-0.002$ & 0.46 & $0.30+/-0.04$ & 0.9945 \\
\hline 7 & $0.0004+/-0.0001$ & $0.78+/-0.03$ & $0.010+/-0.004$ & 0.46 & $0.30+/-0.05$ & 0.9897 \\
\hline
\end{tabular}

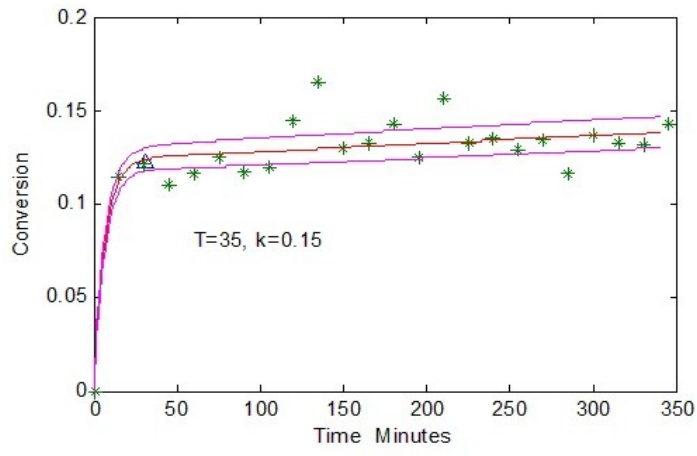

(3a)

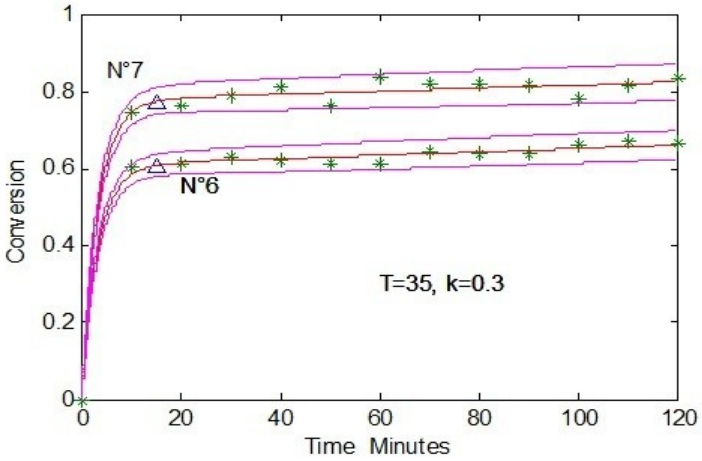

(3b)

Fig. (3). (a-b). Effluent from the textile industry treated under the experimental conditions of Table 3. The triangle marks the estimated time to reach a plateau obtained by Equation 18.

Table 3. Experimental Conditions Analyzed for the Effluent from the Textile Industry

\begin{tabular}{|c|c|c|c|c|c|c|}
\hline $\mathbf{N}^{\circ}$ & $\mathbf{U V}$ & $\mathbf{p H}$ & $\mathbf{T}\left({ }^{\circ} \mathbf{C}\right)$ & $\mathbf{H}_{2} \mathbf{O}_{2}$ & $\mathbf{F e}^{+2}$ & 18 \\
\hline \hline 1 & 1 & 5 & 35 & 15 & 18 & $\mathbf{O}_{3}$ \\
\hline 2 & 0 & 5 & 35 & 15 & 18 & 2 \\
\hline 3 & 1 & 5 & 35 & 15 & 4 \\
\hline
\end{tabular}

Table 4. Parameters of the Model Obtained from the Experimental Conditions Analyzed for the Effluent from the Textile Industry

\begin{tabular}{|c|c|c|c|c|c|c|}
\hline $\mathbf{N}$ & $\mathbf{a}$ & $\mathbf{b}$ & $\mathbf{c}$ & $\mathbf{p}$ & $\mathbf{k}$ & $\mathbf{R}^{2}$ \\
\hline \hline 1 & $0.00004+/-0.00003$ & $0.124+/-0.008$ & $0.015+/-0.002$ & 0.46 & $0.15+/-0.007$ & 0.8371 \\
\hline 2 & $0.0000+/-0.0005$ & $0.15+/-0.03$ & $0.019+/-0.005$ & 0.46 & $0.15+/-0.004$ & 0.8878 \\
\hline 3 & $0.0002+/-0.0001$ & $0.26+/-0.02$ & $0.029+/-0.006$ & 0.46 & $0.15+/-0.004$ & 0.9785 \\
\hline
\end{tabular}

and $\mathrm{Fe}^{2+}$ presented in 6 and 7 in the Table 2, i.e., the degradation reaction remains significant after reaching the level estimated by the time $t_{\text {pat }}$ in Equation 18.

Finally, the results for effluent from the textile industry, treated in three experimental conditions, are shown in Table 3 and the estimated parameters in Table 4. The first point highlighted is the parameter $p$ value of 0.46 , a figure very similar to those found in the modeling for the first effluent.
This result provides evidence that this parameter can be approximately 0.46 for various types of treated effluents by AOPs.

Data from the degradation reaction in experimental condition 1 were obtained in a time interval greater than in other experimental conditions analyzed in this work. This allowed an evaluated behavior of the reaction in the plateau for a longer duration, as seen in the Fig. (3a). Again the confi- 
dence intervals highlighted points with considerable noise and/or experimental errors in their measurements, deviating significantly from the average behavior. For this effluent, the parameter $b$ must be highlighted as it was obtained in experimental condition 3 and showed notably elevated values than those found in conditions 1 and 2. This is shown in the combined action of ozone and UV in the degradation process as presented in Fig. (3b).

\section{CONCLUSIONS}

With the stochastic equation presented, it was possible to model the average behavior and the variability in degradation of two different types of industrial effluents treated by different oxidation processes using only five stochastic model parameters dependent on the experimental conditions in the degradation process. An interesting result is that the parameter $p$, related to data scattering modeling, showed a value close to 0.46 , for both effluents studied under different experimental conditions. Tt was possible to identify experimental data with strong chemical interference and/or experimental error in their measurements using confidence intervals for COD conversion. Finally, based on the model, a simple formula was obtained to estimate the reaction time to reach a plateau.

\section{CONFLICT OF INTEREST}

The authors confirm that this article content has no conflicts of interest.

\section{ACKNOWLEDGEMENTS}

FAPESP $N^{0}$ 2009/17650-2 process.

\section{REFERENCES}

[1] H.S. Costa, and L.A. Daniel, "Experimental Study Using Ozone: applicability of DPD Method and interference in analytic methods for hydrogen peroxide" Rev. De. Ciência. Tecnol., vol. 12, pp. 3947, 2007.

[2] R. Durrett, Stochastic Calculus: A Practical Introduction, CRC Press LLC: Florida, 1996.

[3] B. Efron, and, R.J. Tibshirani, An Introduction to the Bootstrap, Chapman \& HallL: New York, 1993.

[4] P.R. Gogate, and A. Pandit, "A review of imperative technologies for wastewater treatment I: oxidation technologies at ambient conditions", Adv. Environ. Res., vol. 8, pp. 501-551, 2004.

[5] L. Kelly, E. Platen, and M. Sørensen, "Estimation for Discretely Observed Diffusions Using Transform Functions", J. Appl. Probab., vol. 41, pp. 99-118, 2004.

[6] F.C. Klebaner, "Introduction to the Stochastic Calculus with Applications", Imperial College Press: UK, 2008.

[7] L.C Lange, J.F. Alves, M.C.S. Amaral, and W.R. Melo Júnior, "Sanitary landfill leachate treatment by fenton oxidation", Eng. Sanit. Ambient., vol.11 , pp. 175-183, 2006.

[8] E. Neyens, J. Baeyens, "A review of classic Fenton's peroxidation as an advanced oxidation technique", J. Hazard. Mat., vol. 98, pp. 33-50, 2003.

[9] M. C. Oliveira, R.F. Pupo Nogueira, J.A. Gomes Neto, W. F. Jardim, J.J.R, "Rohwedder, Sistema de injeção em fluxo espectrofotométrico para monitorar peróxido de hidrogênio em processo de fotodegradação por reação de foto-Fenton", Química. Nova., vol 24, no. 2, pp.188-190, 2001.

[10] Peixoto, André Luís de Castro; Brito, Renata Alves de; Salazar, Rodrigo Fernando dos Santos; Guimarães, Oswaldo Luiz Cobra; Izário Filho, Hélcio José, "Prediction of chemical oxygen demand in mature landfill leachate doped with Fenton's reagent, using empirical mathematical model obtained by full factorial design", Química. Nova., vol. 31, no. 7, pp.1641-1647, 2008.

[11] P. Kloeden, and E. Platen, Numerical Solutions to Stochastic Differential Equations. $3^{\text {rd }}$ ed. Springer-Verlag: USA, 1997.

[12] Rodryguez J, A, Ovejero, G, Romero, M.D. Dyaz, C. Barreiro, J. Garcya, "Catalytic wet air oxidation of textile industrial wastewater using metal supported on carbon nanofibers", J. Super. Crit. Fluids., vol. 46, pp. 163-172, 2008.

[13] E.C.G Rufino, and L.A. Faria, "Degradação de corantes têxteis através de ozônio gerado eletroquimicamente sob eletrodos de B$\mathrm{PbO}_{2}$ ", Horizonte. Científico., vol. 1, p. 9, 2007.

[14] D.M. Tobaldi, A.Tucci, G. Camera-Roda, and G. Baldi, L. Esposito, "Photocatalyticactivity for exposed building materials". J. Eur. Ceram. Soc., vol. 28, pp. 2645-2652, 2008.

Received: May 17, 2011

Revised: July 13, 2012

Accepted: July 18, 2012

(C) Siqueira et al.; Licensee Bentham Open.

This is an open access article licensed under the terms of the Creative Commons Attribution Non-Commercial License (http://creativecommons.org/licenses/by-nc/3.0/) which permits unrestricted, non-commercial use, distribution and reproduction in any medium, provided the work is properly cited. 